\title{
OSGOOD ON ANALYTIC FUNCTIONS OF SEVERAL VARIABLES
}

Lehrbuch der Funktionentheorie. By W. F. Osgood. Part I. Leipzig, B. G. Teubner, 1924. v+242 pp.

It may appear strange to give a review of a part of a volume, an "Erste Lieferung." There is, however, good justification for this. The present 242 pages make a substantial unit by themselves. They comprise the general concepts and theorems which form the basis for a general theory of analytic functions of two or more variables. Subsequent chapters to be added later will be devoted to special branches from the main trunk, such as the theory of $2 n$-ply periodic functions of $n$ variables, theta-functions, or automorphic functions in more than one variable; and so on. Since also some considerable time will elapse before the writing and publication of these chapters, it seems both suitable and desirable to review the present brochure.

The need of an up-to-date coordination and systematization of material relating to analytic functions of several variables is almost too apparent to enlarge on. While many of the results and methods for analytic functions of one variable can be carried over without difficulty to functions of many variables, the difficulties at other points are so great, the pitfalls so hidden, the progress so slow, as to make it almost legitimate to say that a profound gulf exists between the theory of analytic functions for a single variable and that for several variables.

The literature pertaining to analytic functions of several variables is rather scattered, and little in the nature of systematic exposition is to be found. Chapter XVII in Goursat's Cours d'Analyse and Chapter IX in the second volume of Picard's Traité d'A nalyse are excellent so far as they go, but are confined to the elementary aspects of the subject. Then there are the Calcutta lectures of Forsyth upon Functions of Two Complex Variables (1914), but so different are they in character, scope, and content from the work under review that they increase the desirability and usefulness of the latter. Synoptic reports on analytic functions of many variables are to be found in fourteen pages of the section written by Osgood a quarter-century ago for the Encyklopaedie der Mathematischen Wissenschaften, in Hartogs' Beright in the Jahresbericht der deutschen Mathematiker-Vereinigung (vol. 16 (1907)), and finally in Bieberbach's recent encyclopaedia article (1921), giving the Neuere Untersuchungen über Funktionen von komplexen Variablen, of which, however, only a tenth is devoted to analytic functions of several variables. Lastly, I may cite as a forerunner of the present work the Madison Colloquium Lectures of Osgood (1914), treating Topics in the Theory of Functions of Several Complex Variables. Naturally, these lectures have also largely the nature of encyclopaedic sketches or skeleton outlines without the connective tissues.

Such a meager amount of literature reveals how strangely little the analytic function-theory has been in fashion mathematically, although its 
fundamental importance is undeniable. One of the reasons for this is doubtless its difficulty. The task before Osgood was no easy one: to find out where the known stops and where the unknown begins, to observe carefully the limitation upon the theorems for the case of $n$ variables, to ascertain and weigh the value and significance of the newer methods which have been beaten out where the older methods collapsed. I have often wished for a place in mathematical literature where one could find a comprehensive, connected, and properly restricted modern exposition of analytic functions of several variables, a spot upon which one could plant his feet as on a rock. At last we have it!

Osgood's material is divided into three chapters: Chapter 1. Integraldarstellungen und mehrfache Reihen. Die erweiterten Räume. Chapter 2. Implizite Funktionen. Teilbarkeit. Chapter 3. Singuläre Stellen und analytische Fortsetzung. Rationale Funktionen.

The first chapter, for the most part, containsthe theorems which can be obtained by ready extension of the theory for one variable. For this purpose two standard methods are available, the one generalizing Cauchy's integral, and the other using the power series of Weierstrass. Correspondingly, the phrases "Integraldarstellungen" and "Mehrfache Reihen" appear in the title of the chapter. Consistently with volume I, an analytic function $f\left(z_{1}, \cdots, z_{n}\right)$ is defined as a function which is onevalued at every inner point of a region $T$ and has throughout the region a partial derivative with respect to each of the $n$ variables. The continuity of the function is rightly assumed at the start. In the third chapter, as a much later development, it is shown how this assumption of the continuity of $f\left(z_{1}, \cdots, z_{n}\right)$ can be removed. The removal was first effected by Osgood when the function is bounded, and later Hartogs succeeded in removing even this restriction.

To treat analytic functions im Grossen their behavior at infinity must be considered. Accordingly, it is necessary to explain how the $n$-fold complex space of the variables $z_{i}$ or the equivalent real space $\left(x_{1}, y_{1}, \cdots\right.$, $x_{n}, y_{n}$ ) of $2 n$ dimensions is to be extended by the invention of points and configurations at infinity ("Die erweiterten Räume"). One of the admirable features of the book is the treatment of this matter. The reader may recall how Bôcher (this Bulletin, vol. 20 (1914), p. 185) has emphasized that there were other ways than the conventional one of extending space and that the object in view must determine the character of the spacecompletion "at infinity." Osgood re-emphasizes this view by indicating several possible extensions for the space of the $n$ variables of our analytic functions. The usual extension gives what the author fittingly calls the "space of analysis," and is obtained by adding a single point in each of the $n$ complex planes of our $n$ variables $z_{1}, \cdots, z_{n}$. In consequence, the infinite region consists of all points $\left(z_{1}, \cdots, z_{n}\right)$ for which one or more of the $n$ coordinates is infinite. Another kind of space, which I shall refer to as complex projective space, results by basing the extension upon the familiar linear projective group in $n$ variables (allowed to be complex), and this has been fruitfully employed by Osgood himself. 
The second chapter is rooted in Weierstrass's so-called preparation theorem, with which it opens. Upon this as a foundation is built the theory of implicit analytic functions, and another application is to the concept and theory of reducibility (Teilbarkeit). The former of these two topics is treated with great fullness, as we would wish, since the field of implicit functions has been especially studied in America by Osgood and his former pupils, Dederick, Clements, etc., and also by Bliss. Systems of simultaneous analytic equations and parameter representation im Kleinen are also considered.

In the third chapter the subject of singular points and configurations is first introduced, and it is devoted in large part to an exposition of the theorems of Hartogs, Levi, Cousin, etc., which treat of analytic behavior, meromorphic behavior, etc., im Grossen. It has the special interest of bringing to the reader the modern methods devised to establish recalcitrant theorems which, though perhaps long surmised, have occasioned great diffculty and ofttimes have been demonstrated either restrictedly or inadequately.

This division of contents is very natural, and altogether logical. But there is so much material to pack away as to give trouble in sectional subdivision - at least, it seems so to the reviewer. One finds a certain lack of swing and natural evolution, particularly in the first two chapters. This seems to me to be the one and only marked deficiency of the book. To apply a trite phase, the trees in the wood are so thick that the map is easily lost. The reader will, I think, find it helpful to keep on hand Osgood's Colloquium Lectures, and use it as an aid in getting a bird's eye view over the whole. No one, however, can fail to feel the difficulty of organizing so much material nor be unaware that the parts fit together, like the stones of a grand mosaic, to make one great design.

The richness of the material is a constant delight. It is impossible to read without perceiving the tremendous amount of study which must have been spent in collating and correlating, and without prizing the thoroughness and completeness with which this has been done. Further, the character of the analytic function theory for two or more variables is such as to demand scrupulous care, and we are glad that the task has fallen to an Osgood. We congratulate ourselves that he did not conventionally stop with the analytic theory of a single variable.

The successive topics are developed very fully. In Chapter I there is frequently a rapid change from one topic to another having little relation to it. To cite only a single instance, the section on homogeneous coordinates is succeeded by one on Laurent's series, and this is followed by one on analytic continuation. The section on Laurent's series is thus sawed off from other work on series. The matter is, however, very closely interwoven. If one attempts to select and study some particular topic here or there, he will usually find it imperative to go back and digest something that has gone before, and from this he may have to turn to something still further back. There is also continual reference by chapter and section numbers to volume I. Osgood presupposes justly that the reader should already have a firm grasp on the function theory for one 
variable. The book is not one to be used to get an understanding of function theory for one variable from that for $n$ variables by putting $n=1$. One must be content to proceed slowly and to think and ponder as he proceeds. When he has so done, he will be well satisfied with the grip obtained.

I turn now to a more detailed notice of the features of Chapter I. After a variety of topics, such as the Differential, the Mittelwerthsatz, Liouville's Theorem, and certain work on implicit functions, he paves the way for the all important Cauchy integral theorems. In $\$ 8$ we find the needed theorems regarding the analytic character of the integral

$$
\int_{C_{1}} d t_{1} \int_{C_{2}} d t_{2} \cdots \int_{C_{m}} f\left(t_{1}, t_{2}, \cdots, t_{m} ; z_{1}, \cdots, z_{n}\right) d t_{m},
$$

where $f$ is continuous in its $n+m$ arguments for values of the $t_{i}$ on "regular" curves $C_{i}$ in the $t_{i}$-planes and for values of the $z_{i}$ in $n$ regions of the complex plane of these $n$ variables, and furthermore is analytic in the $z_{i}$ for these values of the arguments. In $\$ 9$ the form of the region is specified to which the work is subsequently largely confined. This is what he terms a cylindrical region (Zylinderbereich) in the space $S_{2 n}$ of $2 n$ dimensions which is obtained by associating arbitrarily the values $z_{i} \equiv x_{i}+i y_{i}$ belonging to the inner points of $n$ independent regions (not necessarily simply connected) in the $n$-planes of the separate variables. The care of the author in specializing the regions he uses and thus preventing his reader from mentally extending conclusions to any old kind of region is a laudable characteristic of the book. After the introduction of Cauchy's integral formula for $f\left(z_{1}, \cdots, z_{n}\right)$ and the deduction from it of various important consequences, the scene shifts to a rather uninteresting analogue of the mean value theorem of the differential calculus and then to a nineteen page discussion of "Mehrfache unendliche Reihen," which by the definition are absolutely convergent. For power series $P\left(z_{1}-a_{1}, \cdots, z_{n}-a_{n}\right)$, it is noted that the region of absolute convergence may consist not merely of the simply connected region of $2 n$ dimensions usually considered, but also, possibly, of regions of $2 k$ dimensions $(k<n)$ in the coordinate planes. I mention this casually to illustrate thereby the variety of interesting and minor features which arise from the author's care. The sections on series culminate, of course, in the familiar Cauchy-Taylor power expansions in $n$ variables. In the sections next following, the space for the analytic functions is extended appropriately to infinity, first without the introduction of homogeneous coordinates. Then, after their introduction, he goes on to establish that in the resulting $2(n+1)$-fold space of the new homogeneous variables corresponding to the space $S_{2 n}$ of the original variables $z_{i}$, the function will be analytic or meromorphic in terms of the new $n+1$ homogeneous variables if it is so in the corresponding space of the original $n$ variables. We are next surprised by the remarkable theorem (p. 57) that if $f(w, z)$ is analytic and finite for the region $|w|+|z|>G$, where $G$ denotes a fixed number, then $f$ is a constant. This theorem is one of few which is without an analogue for the analytic function of one variable. The subject of analytic continuation of functions by successive overlapping regions is, of course, conventional, but in considering analytic extension along a regular curve in 
$S_{2 n}$ the author takes the pains to introduce a specific form of region both for extended and unextended space to take the place of the circle of convergence used in analytic extension along a curve in the complex plane of a single variable, and this is done both for the space of analysis and for complex projective space.

The first three sections of Chapter II relating to the so-called preparation theorem of Weierstrass are particularly good, just as we would wish at so important a place. For the sake of what is attached to it, I repeat the theorem here: If an analytic function $F\left(w, z_{1}, \cdots, z_{n}\right)$ vanishes at a point $\left(b, a_{1}, \cdots, a_{n}\right)$-for convenience taken to be $\left(0, a_{1}, \cdots, a_{n}\right)$ -but without vanishing identically around it, then in an appropriate vicinity, $|w|<r,\left|z_{j}-a_{j}\right|<h,(j=1, \cdots, n)$, we can express $F$ in the form $F\left(w, z_{1}, \cdots, z_{n}\right)=\left(w^{m}+A_{1} w^{m-1}+\cdots+A_{m}\right) \Omega\left(w, z_{1}, \cdots, z_{n}\right)$,

where the $A_{k}$ are functions of $z_{1}, \cdots, z_{n}$, which are analytic and vanish at the point $\left(a_{1}, \cdots, a_{n}\right)$, while $\Omega$ is a function which does not vanish in the vicinity specified. The case in which $F\left(w, a_{1}, \cdots, a_{n}\right)$ vanishes identically is first discharged in the manner of Weierstrass by an appropriate linear transformation in the $n+1$ variables $w, z_{1}, \cdots, z_{n}$, and then Osgood goes on to prove that the form above given to the preparation theorem will hold also in that case for $n=1$, if we insert before $w^{m}$ in the above formula a coefficient $A_{0}(z)$ which vanishes at $z=0$. By means of a special example Osgood shows further that a like form will not hold for $n>1$. This failure to hold is just one of many instances showing how extremely careful one must be in generalizing the ordinary analytic theory for one independent variable.

The first factor in the above preparation theorem brings before the reader an instance of what the author calls a pseudo-polynomial, that is, an expression $a_{0} w^{m}+a_{1} w^{m-1}+\cdots+a_{m}$ in which the $a_{k}$ denote functions $a_{k}\left(z_{1}, \cdots, z_{n}\right)$ which are all analytic at some point $\left(c_{1}, \cdots, c_{n}\right)$ under consideration, called the Spitze of the pseudo-polynomial. The existence of the pseudo-polynomial in the above preparation theorem suggests the possibility of further decomposition of the analytic function into pseudopolynomial factors. We are thus led to the theories of divisibility, reducibility, resolution into prime factors, etc., which follow. It may be remarked that the theories are almost altogether "im Kleinen." By equating the pseudo-polynomial to zero we are led to implicit function theory and also to a study of functions on the resulting pseudo-algebraic "Gebilde." A considerable part of this second chapter is rather specialized work on implicit function theory, the Jacobian, Riemannsche Räume generalizing Riemannian surfaces, systems of pseudo-algebraic equations, etc. For a first reading, one can well pass over a large portion of this chapter, confining his attention after $\$ 10$ to the parts relating to functions on a pseudoalgebraic Gebilde and to parameter representation, and proceed directly to Chapter III. Indeed, we rather regret that some of the material was not postponed to a Chapter IV, so that first essentialscould be more rapidly reached and picked out.

Chapter III compels one's interest. In the opening section, the author first discusses the topic of the singularity of the analytic function. The 
chapter deals chiefly with the unessential singular point (ausserwesentliche singuläre Stelle), in the vicinity of which the function can be expressed in the form $G\left(z_{1}, \cdots, z_{n}\right) / H\left(z_{1}, \cdots, z_{n}\right)$, where $G$ and $H$ are both analytic and the latter vanishes at the point. When the numerator does not vanish, we get an unessential singularity of the first kind, which is the analogue of the familiar pole, and when it vanishes, an unessential singularity of the second kind, which much resembles the essential singularity of an analytic function of the single variable. When all the singularities in a given region are unessential, the function is, of course, called meromorphic.

Some of the greatest difficulties in the study of analytic functions of many variables arise from the fact that the zeros and singularities are never isolated, but form a continuous configuration. Thus, unessential singularities of the first kind fill the $(2 n-2)$-fold manifold $H\left(z_{1}, \cdots, z_{n}\right)$ $=0$, and those of the second kind (in whose vicinities the function is unbounded) fill a $(2 n-4)$-fold manifold. Not only are these (obviously) excluded in the two remarkable theorems due to Kistler, which the author goes to prove, but also all other singularities. The first of these theorems is as follows. If a function $F\left(z_{1}, \cdots, z_{n}\right)$ is analytic in a $2 n$-dimensional region $T$ except possibly for the points of a $(2 n-2)$ fold manifold and is furthermore limited, then the function can be continued analytically over the entire region $T$, so that the points of the exceptional manifold, if singular, are removable discontinuities. The second theorem is like the first except that the possible exceptions make at most a $(2 n-4)$-fold manifold, while the restriction that the function shall be limited is altogether omitted. The method of proof of these theorems and of others consists mainly of adroit use of Cauchy's integral formula. The same tool gives a number of very interesting theorems, due for the most part to Hartogs, which continue a function analytically beyond the region of its analytic definition. These culminate in Hartogs' fine result that a function $f\left(z_{1}, \cdots, z_{n}\right)$ which is one-valued and analytic on the boundary of a "regular" cylindrical region permits of analytic extension over the whole interior of the region. This conclusion is noteworthy inasmuch as the corresponding statement is not true in the case of a function of a single variable. After the theorems of Hartogs come the noteworthy theorem of E. E. Levi relating to meromorphic continuation of an analytic function. In particular, the theorem of Hartogs last cited will continue to hold if the word "analytic" is replaced by "meromorphic."

These results can only hint at the wealth of interesting theorems in this third chapter of Osgood's work. We find in accessible form Cousin's extensions of the well known theorems of Weierstrass for the representation of an entire function as a product of factors exhibiting zeros, and of MittagLeffler for the representation of a meromorphic function with arbitrarily distributed poles. The extension of the former given by Cousin contained a vitiating error, in that Cousin did not properly delimit the connectivity of the region. Gronwall, who started on the consideration of analytic functions of several variables in his Uppsala thesis, later rendered an 
important service in detecting the hidden error and properly restricting the result.

Finally, we reach the theorem that a function meromorphic in a simply connected region (which may be the entire finite space) can be expressed as the quotient of two functions analytic over the region. This is established by Osgood without the use of the harmonic functions of many variables employed by Poincare in the first demonstration given, the theory of which is even today without systematic development. Then follows the Weierstrass-Hurwitz theorem that a function meromorphic over the entire plane is a rational function. Special attention may be called to the remarkable fact that the theorem is not merely valid for "the space of analysis", but was proved by Osgood to hold also for complex projective space (and even for space extended in a mixed manner to infinity (Jackson)). I can not forbear to mention the simple proof that every one-to-one analytic* transformation of the extended space of analysis into itself has the form

$$
z_{i}=\frac{a_{k} z k+b_{k}}{c k z k+d_{k}}, \quad(i=1, \cdots, n),
$$

where the $n$ values of $k$ appearing in the right-hand members are the numbers $1,2, \cdots, n$ in arbitrary order. This theorem is well known for the case of a single variable $z$, though even for this case the reader may experience trouble in ascertaining where a demonstration is to be found. References to literature are abundantly given by Osgood, but at this point, as at some others, we find none at all. We have the suspicion, therefore, that the general proof here given is the authors' own, as well as a corresponding theorem for complex projective space.

The volume is by no means easy to read, but grows simple if one delves and ponders. It shows the same thorough, massive scholarship, extending to all details, which I commented upon so favorably in my review of volume I. If we deplore again that the book could not have been written in English, we recognize a certain fitness that it appears in German, for it displays the inspiration of a German graduate training, as well as the persistent, accurate scholarship so characteristic of German work.

The author wishes me to call attention to the following corrections. Page 129, line 4 from the top. Reference should be to the Beispiel von \$2 (not \$22). Also in the middle of the page read $2(y / x)$ in place of $2(y / z)$. Page 33, line 3 from bottom. Instead of $a_{k} \neq 0, k=1, \cdots, n$, read $\sum\left|a_{k}\right|>0$, $(k=1, \cdots, n)$.

\section{E. B. VAN VLECK}

* Of course, with proper modification in the infinite region. 